Voix et Images

voixetimages

\title{
La problématique nationaliste dans l'oeuvre romanesque de Jacques Godbout
}

\section{Jacques Pelletier}

Volume 6, numéro 3, printemps 1981

Philippe Haeck

URI : https://id.erudit.org/iderudit/200284ar

DOI : https://doi.org/10.7202/200284ar

Aller au sommaire du numéro

Éditeur(s)

Les Presses de l'Université du Québec

ISSN

0318-9201 (imprimé)

1705-933X (numérique)

Découvrir la revue

Citer cet article

Pelletier, J. (1981). La problématique nationaliste dans l'oeuvre romanesque de Jacques Godbout. Voix et Images, 6(3), 435-451. https://doi.org/10.7202/200284ar d'utilisation que vous pouvez consulter en ligne.

https://apropos.erudit.org/fr/usagers/politique-dutilisation/ 


\title{
La problématique nationaliste dans I'ceuvre romanesque de Jacques Godbout ${ }^{1}$
}

\author{
par Jacques Pelletier
}

Dans la seule étude d'ensemble - à ma connaissance - consacrée à ce jour à la production romanesque de Godbout. André Smith formule l'hypothèse que cette œuvre est d'abord et avant tout, sinon exclusivement, une variation sur le thème de l'amour et de la séparation. «De L'Aquarium à $D^{\prime}$ Amour P.Q. écrit-il, seules les apparences changent. Pour l'essentiel, le narrateur nous raconte invariablement I'histoire d'un couple qui n'arrive pas à se former ${ }^{2}$. Cela dit, bien entendu, le critique n'a pas pu faire autrement que de constater qu'il y avait aussi dans ces romans une dimension politique explicite. Cependant, dans sa perspective, cette dimension politique est logée à l'enseigne de ce qu'il appelle le «récit secondaire » des romans de Godbout par opposition au récit principal: I'histoire des amours contrariées - axe narratif qu'il estime «par définition tributaire de ce dernier - l'axe du discours amoureux - qu'il a pour fonction de masquer " et ce en dépit du fait reconnu que «le récit secondaire occupe beaucoup plus d'espace que le récit principal dans l'ensemble du texten ${ }^{3}$.

Par la suite, conséquent avec son hypothèse et son approche méthodologique relevant de la tradition psycho-critique, Smith tente de montrer qu'une relation amoureuse est au centre de chacun des romans. Même à ce point de vue, on pourrait faire remarquer que sa démonstration pose problème. Car s'il est incọtestable qu'un roman comme Le couteau sur la table se présente d'abord comme mise en scène d'un drame amoureux, il n'en va pas de même évidemment pour les quatre autres romans. Ceci dit, là n'est pas l'essentiel. Ce qui doit plutôt retenir notre attention, c'est l'affirmation suivant laquelle ce qui est «secondaire" - la dimension politique des romans - occuperait néanmoins plus d'espace dans le texte que ce qui constitue le «principal» le discours amoureux. Si, à la suite de Goldmann, on adopte comme critère de validité et de pertinence d'une lecture son pouvoir de rendre compte de la totalité, ou a tout le moins de la plus grande partie, d'un texte, il me semble que l'hypothèse de Smith doit être questionnée et qu'il est possible d'en proposer une autre. Dans cet ordre d'idées, il apparaît que ce qui structure cette cuvre exception faite de I'/s/e au dragon - c'est bel et bien la dimension écartée comme inessentielle par Smith, le politique, et plus précisément l'idéologie nationaliste. 
C'est l'hypothèse que j'entends développer dans cet article d'une part au moyen d'une étude interne des romans de Godbout et, d'autre part par leur mise en rapport avec une conjoncture (et son évolution) littéraire, idéologique et politique, le principe d'analyse étant, dans cette seconde étape, qu'une cuvre se développe et se situe dans un contexte précis, d'abord dans le champ littéraire, puis de manière plus large dans le champ idéologique et entin, avec plus ou moins d'intensité selon les périodes, dans le champ plus englobant du politique (entendu au sens large, incluant donc l'économique et le social). Cette méthodologie, par ailleurs, renvoie à une conception de l'œuvre littéraire comme production d'un individu, avec les déterminations psychologiques et sociales qui s'exercent sur lui, dans une conjoncture historique possédant également ses déterminations concrètes.

\section{La recherche d'identité comme principe de structuration romanesque}

\section{A) Des romans du prob/ématique...}

A première vue, I'Aquarium, le premier roman de Godbout, écrit au tout début des années 1960 et publié en 1962, apparaît étranger à la problématique nationaliste. D'une certaine manière, on pourrait, en caricaturant à peine, le «résumer * de la façon suivante: un héros, jeune coopérant dans un pays colonisé, raconte ses états d'âme - son mal de vivre et ses désillusions - exacerbés par la mort du seul homme qu'il admirait dans l'univers des colons auquel il appartient, rumination morose dont la venue de l'ancienne maîtresse du disparu le débarrassera en provoquant chez lui la prise de décision - et la volonté - de quitter l'univers, l'aquarium poisseux, dont il est prisonnier.

Lu selon cette optique, le roman constitue une variation canadienne française sur les thèmes majeurs de l'existentialisme, alors courant dominant dans le champ littéraire français. Le héros se débat en effet avec les angoissantes questions de la solitude, des raisons de vivre et/ou de mourir, du sens (ou non) de l'histoire, de l'engagement, etc. Sur toutes ces questions, il se montre irrésolu, velléitaire: anti-conquérant en somme, contre-modèle des héros agissants fascinés par la puissance d'un Malraux. Cependant cette «impuissance» du héros n'apparaît pas par hasard et n'importe où: elle naît d'un contexte précis dont, à sa manière, elle fournit une image. Et, contrairement a ce que soutient Smith, la relation essentielle du roman n'est pas celle du héros et de l'ancienne maîtresse du disparu, mais bien celle, beaucoup plus centrale - et problématique - du héros et de la communauté.

II se dégage en effet nettement que le roman raconte le «drame» d'un héros coincé entre deux univers: celui des colonisateurs, jugé décadent et menacé, celui des colonisés, fait de promesses, ouvert sur l'avenir mais auquel il est impossible de s'identifier totalement. Seule issue possible à ce déchirement: la fuite au terme d'une prise de conscience par le héros de ce qu'il est: un petit bourgeois agnostique, ne croyant à aucune valeur absolue (pas même à la *révolution" qui avait pourtant motivé son engagement comme coopérant et qui, lorsqu'elle advient pour de vrai, lui fait peur). Andrée, dans 
cette perspective, joue un rôle de révélateur et de catalyseur: son arrivée précipite la prise de décision du héros, mais ne la crée pas: elle s'imposait de toute manière étant donné le caractère "contraignant» en quelque sorte de la situation de base du récit.

Le héros, donc, est coincé entre deux univers: celui des coloniaux, monstre à deux têtes en période de décolonisation: la nouvelle élite indigène, le *Cartel féodal Clergé-État ${ }^{4}$ et ses alliés occidentaux, anciens colonisateurs et coopérants ${ }^{5}$; celui des colonisés, des exploités de l'ancien et du nouveau régime: «étudiants, jeunes avocats, quelques ouvriers spécialisés ${ }^{6}$ que représente Gayeta, figure symbolisant l'avenir. Plus précisément le héros appartient à l'univers des coloniaux «étrangers», ces individus attirés par l'aventure et l'argent, ces «importés, exilés volontaires, involontaires, des fuyards " ${ }^{7}$ qui ne croient plus à rien, s'ennuient à mourir, cherchent à noyer leur ennui dans l'alcool et songent vaguement et mollement à repartir: des «vieillards ${ }^{8}$, des $\propto$ escargots ${ }^{9}$ qui végètent à la Casa Occidentale, cet immeuble qui $*$ craquelle $* 10$, ce lieu de décomposition et de décadence où l'on vit «au ralenti»11, où l'on se désintègre lentement mais sûrement faute de croire à des valeurs pouvant justifier l'existence : «Nous avons épuisé tout sujet depuis si longtemps. Nous nous sommes tout dit; d'abord parce qu'il est des mots que nous avons bannis de toute conversation: dignité, justice, liberté -comme s'il s'agissait de fruits exotiques qui n'ont pas leur place dans un plateau de ce salon»12 La Casa - cet aquarium dans lequel marinent de bien misérables poissons - est ainsi un microcosme du régime colonial à son déclin (régime qui a pourtant connu des heures de gloire quand l'Occident lui-même croyait a son destin et à la pérennité de sa civilisation, mais ce n'est plus le cas...).

Si le héros appartient, par son origine, par son statut, à l'univers des coloniaux, il reste qu'il ne s'y identifie pas totalement. Contrairement à la plupart des membres du groupe, il a une conscience - un sur-moi - hyperdéveloppée qui le pousse à se sentir «responsable de tout»13, conscient des contradictions dans lesquelles la communauté à laquelle il appartient objectivement se trouve, conscience qui lui permet de porter sur elle un regard critique - il en est tout en n'en étant pas -; en témoignent sa description ironique de l'Église et des prêtres, voleurs comme Monsignore et/ou pédés comme Pauline ${ }^{14}$ et son évocation, sur le mode parodique, de la société de la Casa ${ }^{15}$.

Cependant cette lucidité - qui en fait un marginal - dont le héros fait état dans sa description de la société coloniale - cette distance qui lui a gagné des amitiés parmi les jeunes révolutionnaires indigènes - ne suscite pas chez lui de passage à l'acte: tout au long du récit, il appraît irrésolu à la fois face à ses amis révolutionnaires qui l'invitent à partager concrètement un combat qu'il approuve et, de manière plus générale, face à son propre destin: «Moi j'hésite toujours devant vingt sentiers", et ce n'est qu'à la fin du récit qu'il prend enfin une décision, qui est de fuir.

Au terme de cette très rapide analyse, on voit à tout le moins premièrement que le drame amoureux a une importance secondaire contrairement à ce 
que prétend Smith, deuxièmement que la relation du héros à la double communauté des coloniaux et des colonisés constitue la structure de base du récit, troisièmement que, par suite, la problématique de la décolonisation est au cœur de la thématique du roman (bien qu'elle soit abordée en quelque sorte de l'extérieur, hors du contexte québécois), quatrièmement que cette problématique est couplée à une seconde qui n'est pas sans importance, l'existentialiste (problème du sens de la vie, des valeurs, de l'absurbe etc.); par conséquent on peut déjà avancer, il me semble, que l'explication du roman devra être recherchée dans deux directions majeures: la crise des valeurs vécues par les jeunes gens de la petite bourgeoisie intellectuelle à la fin des années 1950, l'éveil du néo-nationalisme québécois au début des années 1960. On y reviendra dans la seconde partie de ce texte.

Si la thématique nationaliste est abordée «de l'extérieur» dans I'Aquarium, le roman témoignant de l'effondrement général du système colonial sans jamais évoquer directement le cas du Québec, il n'en va pas de même dans Le couteau sur la table publié en 1965. Dédicacé aux animateurs de la revue Liberté, ces partisans de la «révolution tranquille" désireux de voir celle-ci adopter un cours plus nationaliste, ce second roman est présenté par son auteur comme «l'histoire d'une rupture», non pas simplement "entre des êtres qui s'aiment $\%$, mais plus fondamentalement avec une certaine tradition littéraire, le roman étant défini comme une expression parmi d'autres de la "francité", c'est-à-dire d'une nouvelle manière de vivre et de penser en français née à l'extérieur du cadre hexagonal français. Conscient de la nouveauté - et du caractère problématique de ces nouvelles manifestations culturelles et littéraires - Godbout précise en outre que son roman, dans cette perspective, est une «approximation littéraire d'un phénomène de réappropriation du monde et d'une culture»16.

Ce prologue est extrêmement important car il situe, et de manière fort explicite, le roman dans un double contexte, celui de l'affirmation du fait national au Québec, celui, plus général, de la décolonisation, et notamment de celles des anciennes colonies françaises. Pour exprimer ces réalités nouvelles, il faudra, et c'est implicite dans le prologue, créer une littérature nouvelle, originale, qui ne soit plus une simple manifestation de la littérature française dans un cadre extraterritorial. Par ailleurs le roman, on le verra tout de suite, s'inscrit également par sa thématique dans la problématique coloniale telle que vécue et exprimée - conceptualisée - au Québec.

Par sa structure, Le Couteau apparaît en continuité directe avec I'Aquarium. À nouveau nous sommes en présence d'un héros-narrateur coincé entre deux univers, celui des coloniaux qui, dans le cadre canadien, sont essentiellement les Canadiens anglais, celui des colonisés, les Canadiens français, généralement pauvres, du Québec. L'opposition entre ces deux univers est incarnée par deux femmes, Patricia et Madeleine, entre lesquelles le cœur du héros balance. Patricia représente le "côté faible» du héros, sa séduction par une femme à l'aise dans sa peau, riche, incarnation par excellence de la civilisation de l'argent qui, à la fois, l'attire et le dégoûte. Celui-ci est en effet, alternativement, fasciné par le bonheur de vivre de Patricia 
et scandalisé par son «irresponsabilité». Madeleine, par ailleurs, symbolise la communauté canadienne-française, étant de l'Est de Montréal mais appartenant toutefois, par ses études, au «prolétariat de luxe» ${ }^{7}$, à une catégorie sociale donc qui, à force de travail et de volonté, pourra éventuellement s'émanciper et s'épanouir. Si les rapports du héros avec Patricia sont faits de fascination et de haine mêlés, avec Madeleine ils sont plutôt faits d'amitié, d'une solidarité créée par le partage en commun d'un destin et d'une condition. Le héros, on le sait, ne choisit pas vraiment entre les deux femmes: durant un moment même il tentera avec elles la cohabitation. Puis Madeleine morte, il volera son employeur et fuira aux U.S.A. et au Mexique avant de revenir à Patricia au terme d'une longue équipée. Finalement, d'une certaine manière, il «choisit» in extremis entre les deux femmes - entre les deux communautés dans les dernières pages du récit, étranglant Patricia au moment des premiers attentats du F.L.Q. en 1963, le lien étant explicitement établi entre le meurtre individuel et les premières tentatives «insurrectionnelles» au Québec.

On voit que cette histoire d'amour, si elle possède son autonomie propre, ne s'inscrit pas moins dans un cadre beaucoup plus englobant qui est celui du rapport des deux communautés ethniques dominantes au Canada. Et le héros, cette fois-ci, contrairement à ce qui était le cas dans L'Aquarium, appartient à l'univers des colonisés; il y a donc un profond renversement de perspectives entre le second roman et le premier mais sur une toile de fond qui est la même: le rapport colonial. La domination coloniale apparaît maintenant sous quatre formes: premierement sous celle de la domination économique et politique des Canadiens anglais, et plus précisément de la fraction riche de cette communauté qui habite Westmount et règne sur Montréal et le Québec entier - c'est /'ennemiprincipal -; deuxièmement sous celle de la domination économique et militaire des U.S.A. ${ }^{18}$, les Américains étant objectivement alliés des Canadiens anglais; troisièmement sous celle de la domination politique de l'Angleterre dans la mesure où le Canada est perçu et défini comme pays britannique - il s'agit ici toutefois d'un ennemi secondaire -; quatrièmement sous celle, au niveau interne de la communauté canadienne-française, de la domination politique et idéologique du clergé et des notables traditionnels, ses alliés, et de la résignation profonde du peuple canadien-français lui-même qui accepte son sort passivement, se contentant de manifester à l'occasion sa révolte sous une forme travestie, masquée, sans signification politique, dans de dérisoires combats de ruelles.

C'est dans cette difficile situation objective que le héros du roman conduit tout a la fois une histoire d'amour et une interrogation angoissante quant à son devenir. Lorsqu'il écrit son récit en 1962-1963, il a environ trente ans et c'est dix ans plus tôt, à vingt ans, qu'il a vécu l'essentiel de sa liaison avec Patricia. D'origine modeste il a tout de même reçu une bonne instruction qui devrait lui permettre d'échapper à sa condition et de «réussir» socialement. Comme le héros de I'Aquarium il appartient par ses préoccupations culturelles et philosophiques à la nouvelle petite bourgeoisie. Comme le premier héros de Godbout il apparaît également comme un individu lucide, conscient de sa condition propre et de celle de son peuple, mais foncièrement irrésolu. 
Bien qu'adulte par l'âge il ne semble pas pouvoir assumer vraiment des choix. Essentiellement tourné vers une enfance dont il a la nostalgie et une fin d'adolescence marquée par sa liaison avec Patricia - qu'il n'a pas dépassée, surmontée puisqu'il revient à celle-ci dix ans plus tard - il se laisse porter, glisser au fil des événements, laissant le destin décider pour lui. S'il sait ce qu'il ne veut pas - la vie quotidienne des bureaux, le 9 a 5 qui tue lentement - il ne sait pas bien ce qu'il veut et il se cherche dans l'angoisse une raison de vivre, hanté par le spectre de l'échec: « J'ai peur, dit-il, de mourir tout à coup, j'ai peur là, au creux du ventre, de crever sans avoir fait un seul geste qui soit humain, sans laisser derrière moi autre chose que moi qui refroidis, moi qui pourris, moi humus dans le roc et la glaise... ${ }^{19}$. Comment ne pas se rappeler le mot de Perken voulant, dans La vie royale, laisser son empreinte - une trace - sur la carte du Siam? Et à Patricia, le héros dira encore : «Nous sommes si fragiles, il y a tant de choses graves dont il faudrait s'occuper, tant de fascismes!"19. Ceci dit, il reste malgré tout foncièrement passif, laissant en quelque sorte sa mauvaise conscience tourner à vide.

Par ailleurs, sur un autre plan, en tant que membre d'une communauté, le héros s'interroge sur son avenir dans la mesure où celui-ci est lié au destin plus large de la collectivité. Le héros, là encore, a conscience de la singularité de son être collectif de «français en Amérique " ${ }^{21}$, de cette étrange condition vécue par les Canadiens français d'être chez eux sans y être, d'être des Américains sans en être tout à fait, conscience qui crée un malaise du même ordre que celui provoqué par le port d'un vêtement trop grand ou trop étroit, mal ajusté, inadéquat. Comment échapper au malheur d'être Canadien français? En luttant pour que les choses changent, nécessité dont le héros ressent l'urgence en arrivant à l'âge d'homme: "Mais j'atteignais l'âge de la majorité et je me surprenais de plus en plus à me préoccuper d'une tâche collective, je parlais des foules, des devoirs avec des envolées électorales 22. Mais entre parler, dire et faire le pas peut être énorme et le héros (et ses amis) ne le franchira/ront pas, le passage à l'acte étant chez eux inhibé par la "maladie" 23 , par une sorte de paralysie, d'épuisement, de stupeur qu'Hubert Aquin a bien décrite comme caractéristique de l'humeur du Canadien français colonisé - sa fatigue culturelle - C'est la génération qui suit celle du héros, celle qui aura vingt ans en 1963, qui sortira enfin de la torpeur de la manière que l'on sait, servant du coup de catalyseur au geste meurtrier de celui-ci.

On voit qu'entre les deux premiers romans il y a continuité, des ressemblances profondes - au niveau formel: la composition par fragments; au niveau de la représentation des héros: jeunes hommes de la aclasse moyenne», ayant fait des études, intellectuels petit-bourgeois tourmentés, mal à l'aise dans leur peau, cherchant fièvreusement à donner une direction (un sens) à leur vie - mais aussi des différences: le «cadre» du roman, dans Le Couteau, est canadien-français, le héros n'appartient plus à l'univers des colonisateurs mais à celui des colonisés et le roman n'est plus considéré par l'auteur comme relevant de la littérature française mais bien d'une nouvelle culture internationale, la francité: en cela aussi, il marque une étape importante. Et si les deux romans peuvent être caractérisés comme «romans 
du problématique», constituant un sous-ensemble dans l'œuvre de Godbout, c'est qu'ils renvoient l'un et l'autre à une conjoncture elle-même problématique, le malaise des héros exprimant à sa manière les ambiguités et les impasses de la situation historique.

\section{B) Aux romans de l'affirmation}

Avec Salut Galarneau, publié en 1967, c'est une deuxième étape qui s'ouvre dans l'œuvre de Godbout, celle des romans de l'affirmation, étape qui s'inscrit cependant à l'intérieur du processus plus général de la recherche d'une identité (individuelle et collective) qui, à mes yeux, sert de dénominateur commun et de principe d'unification de l'ensemble de cette production, à l'exception, en partie tout au moins, de I'/s/e du Dragon.

Sur le plan de la filiation littéraire, on assiste à un nouveau déplacement extrêmement significatif. Du roman de «la francité», cette catégorie aussi vague que générale, on passe au roman québécois placé sous les auspices de Maurice Nadeau, non pas du célèbre intellectuel français directeur des Lettres nouvelles mais bien du «petit gars de Saint-Henri », l'animateur du quartier prolétarien que Godbout a connu à l'occasion du tournage d'un film et qui n'est sans doute pas pour peu dans sa connaissance concrète du Québec réel et sa réconciliation avec le pays.

Sur le plan structurel, il y a aussi des modifications importantes par rapport aux deux premiers romans. Ainsi nous ne sommes plus en présence d'un héros-narrateur coincé de manière radicale entre deux univers antithétiques l'obligeant en quelque sorte à faire un choix. II y a, bien sûr, encore l'univers des colonisateurs, sorte d'édifice à quatre étages avec, au rezde-chaussée, les anglophones riches de Montréal assurant la base de la domination (économique et politique), au premier, les Américains imposant la société de consommation et de gaspillage comme modèle idéologique dominant, au second, les curés en perte de vitesse mais s'accrochant farouchement aux privilèges qui leur restent, au troisième enfin, les Français - ce qui est nouveau mais qui relève pour l'essentiel, on le verra, d'un développement de la conjoncture culturelle et littéraire -, mais cet univers n'est pas incarné dans une personne (comme c'était le cas pour Patricia dans le roman précédent) et il n'est pas cerné de façon aussi nette que dans les romans antérieurs (dans ces espaces symboliques qu'étaient la Casa ou Westmount). De même il $y$ a un univers des colonisés auquel appartient Galarneau, qui n'est pas un jeune bourgeois intellectuel comme les héros précédents mais un "raté" du cours classique devenu vendeur de hot-dogs qui s'est, de ce fait, prolétarisé. Dans ce roman, c'est plutôt Jacques, le frère aîné auteur de textes pour Radio-Canada, qui ressemble trait pour trait mauvaise conscience en moins - aux héros des romans précédents.

S'il y a des déplacements, des modifications, des glissements entre Salut Galarneau et les premiers romans il y a aussi continuité. Le héros principal du récit, à nouveau, est l'objet d'une prise de conscience intérieure qui s'effectue aux niveaux politique et littéraire. 
Sur le plan social et politique, Galarneau comprend peu à peu en suivant l'actualité internationale qu'il y a des pays pauvres et des pays ricies, que les seconds vivent dans une large mesure aux dépens des premiers et que son pays appartient au clan des rapaces, des «salauds» qui exploitent pour leur plus grand profit la misère des autres. Il réalise de même qu'à sa manière il est aussi un exploiteur puisqu'il ne fait concrètement rien pour aider les exploités et cela le révolte ${ }^{24}$. Puis, poursuivant sa réflexion, il se rend compte que s'il est un «exploiteur » involontaire il est aussi une victime "d'une guerre, d'une drôle de guerre qui a dû commencer sans qu'on le veuille comme au Vietnam ${ }^{25}$ : celle de la pénétration, et de la domination, du capital américain au Québec comme - et plus encore que dans le reste de l'Occident. Domination dont sont victimes tous ceux qui n'appartiennent pas aux classes dominantes, et notamment les travailleurs manuels soumis aux aléas d'une conjoncture économique capricieuse sur laquelle ils n'ont pas prise; parlant des maçons qui construisent le mur de ceinture de sa maison, le héros constate que ce sont des «esclaves humains ${ }^{26}$ condamnés au travail occasionnel et au chômage et il se reconnaît en eux dans la mesure où son «métier " à lui vendeur de hot-dogs - n'est guère assuré non plus. Comment échapper à cette condition? En réalisant la reconquête économique du Québec, comme le prêchait alors René Lévesque - l'action du roman se déroulant à la fin de la "révolution tranquille" - auquel il est explicitement fait allusion lorsque Galarneau décide de monter une chaîne de stands de hot-dogs. Le projet, on le sait, avortera - ne devient pas capitaliste qui veut malgré ce que dit le «mythe américain» - et le héros trouvera plutôt sa libération par l'écriture.

La problématique de l'écriture est au centre du récit. Au départ Galarneau n'a pas de vocation d'écrivain particulière. De lui-même il n'aurait jamais songé sans doute à écrire. C'est Marise, sa maîtresse, qui lui suggère l'idée qu'il pourrait éventuellement réussir par l'écriture. Pour celle-ci, qui n'a pas d'instruction, qui lit peu — des journaux populaires, des romans policiers l'écriture, pour quelqu'un qui a «fait des études", devrait être une tâche aisée: elle conseille donc à Galarneau d'écrire un roman policier. Le héros, lui, en sait assez pour savoir qu'il n'est pas facile d'écrire, mais encouragé par l'exemple d'un chauffeur de taxi et d'une matrone retirée des affaires qui ont publié des mémoires, il prend la décision d'essayer, pensant fort prosaïquement que son commerce pourrait probablement profiter de la publicité obtenue par son livre: la conception qu'il entretient de l'écriture, à l'origine, est donc strictement fonctionnelle et utilitaire. Le récit (son récit), dans cette perspective, peut être lu comme l'histoire du passage de cette conception instrumentale de la littérature à une conception plus large de celle-ci comme mode de connaissance du réel et de ré-appropriation de soi, comme l'histoire donc d'une conversion et d'une naissance.

Galarneau, en effet, se prend peu à peu au jeu de l'écriture qui devient pour lui une passion et une raison de vivre. Il lit avec intérêt d'autres auteurs, prête attention à leur style, éprouvant progressivement l'agréable sensation de faire partie de la confrérie. Mais cette activité qui exige beaucoup de temps et d'énergie - on n'atteint pas la jouissance sans peine par l'écriture - 
comporte aussi son revers: elle implique une coupure avec la vie, Galarneau découvrant que «c'est drôle: plus je travaille, plus je me retire, moins je me sens capable de parler, c'est comme si je vivais dans les cahiers»27. Or, pendant qu'il écrit, tout entier absorbé par sa nouvelle passion, il se fait cocufier par son frère Jacques. C'est alors qu'il connaît la tentation de renoncer à la littérature et qu'il décide de faire fortune par le commerce des hot-dogs. Le projet ayant avorté, il se réfugie chez lui et fait construire un mur autour de sa maison pour être à l'abri du monde. Mais le monde, malgré le mur, entre par la télévision notamment et Galarneau, incapable de supporter la solitude, réintègre la société à la fin du récit, ayant résolu comme il dit de récrire désormais, c'est-à-dire de tenter de surmonter la contradiction apparemment irréductible entre la vie et l'écriture, en faisant de la vie de la littérature et vice-versa: un nouvel écrivain est ainsi né à la fin du roman (qui constitue donc l'histoire de sa laborieuse naissance).

Cette naissance, par ailleurs, est représentée comme une allégorie de l'éveil du peuple québécois, le récit de la prise de conscience individuelle (et de la libération) de Galarneau étant une parabole de la prise de conscience collective et de l'éventuelle libération du Québec. C'est en cela que ce roman se présente comme une manifestation littéraire positive et optimiste du néo-nationalisme offensif des années 1960 dont le personnage truculent de Galarneau offre une représentation particulièrement savoureuse; ce héros, en effet, n'est plus un petit bourgeois intellectuel tourmenté et passif, mais un «enfant du peuple» qui vit et agit et dont la santé illustre celle de la 'communauté.

Après un silence de cinq ans, Godbout refait surface en 1972 avec la publication de $D^{\prime} A$ mour P.Q. Sans vouloir anticiper sur une analyse que je développerai plus loin, il me semble important toutefois de souligner que ce roman paraît après la première campagne électorale du P.Q. (avril 1970) et surtout après les événements d'octobre de la même année explicitement évoqués dans le roman qui se situe ainsi dans un contexte politique bien précis. De même la dédicace du livre a Raoul Duguay, alors figure de proue de la nouvelle culture québécoise (américaine et francophone) inscrit ce nouveau roman dans un champ très nettement délimité de l'univers littéraire québécois, à l'intersection de la tradition déjà ancienne du nationalisme et du nouveau courant de la contre-culture. J'y reviendrai.

Sur le plan narratif, le roman comporte une innovation par rapport aux récits précédents en ceci qu'il est porté par trois voix: celle d'abord de Thomas d'Amour, l'écrivain qui lui-même emprunte trois manières, trois styles, fait donc entendre trois paroles, celle ensuite de Mireille, la secrétaire au vert langage chargée d'incarner le parler populaire, celle, enfin, neutre, d'un narrateur hors-champ décrivant' de manière elliptique les gestes des personnages. Cette innovation sur le plan de la narration s'accompagne d'un changement concomitant dans la représentation des personnages: le récit n'est plus essentiellement structuré par la quête d'un petit bourgeois plus ou moins intellectuel, mais bien par le dialogue, la rencontre explosive de deux personnages: Thomas l'écrivain, prolongement des héros antérieurs 
de Mireille, premier personnage de femme à s'imposer dans cet univers essentiellement masculin.

Par ailleurs, sur le plan thématique, $D^{\prime}$ Amour $P . Q$. s'inscrit dans le sillage de Salut Galarneau!, la problématique de l'écriture étant à nouveau au centre du récit. Le héros, cette fois, n'est pas un écrivain «naturel», "populaire » comme Galarneau, mais au contraire un écrivain tout ce qu'il y a de plus traditionnel, style professeur de lettres au département d'études françaises de l'Université de Montréal ${ }^{28}$. C'est de sa conversion en écrivain populaire sous l'influence heureuse de Mireille qu'est faite, pour l'essentiel, l'histoire, l'anecdote du roman est donc à nouveau le récit d'une prise de conscience, d'un passage, d'une transformation qui, en l'occurrence, se déroule en trois temps.

Premier temps: Thomas D'Amour écrit le récit d'une aventure alambiquée se passant dans un pays exotique, dans un temps immémorial, mettant en scène des personnages mystérieux aux noms bizarres: Imroul Kais, Turafa, Manat, etc., aventure dont il apparaît peu à peu qu'elle constitue une version parodique du récit biblique. Cette histoire, par ailleurs, est narrée dans un style ampoulé, précieux, décadent, qui reproduit, de manière ironique, le nouveau roman français. Mireille estime qu'il s'agit d'une sorte de sous-produit de Butor et Mariette, sa copine, commentant un extrait de la préface d'Olivier du Magny à Graal Flibuste de Robert Pinget, dira: "Ça renforce ma théorie: il y en a qui ne font pas l'amour ce sont des écrivains qui gâtent le fonne. La littérature leur reste dans les mains ${ }^{29}$. Mireille ajoutera encore: «C'est clair, ça doit être un texte pour une revue littéraire, c'est sûr... douze abonnés dont dix écrivains et deux professeurs»30, en somme «de la littérature de Haute Classe ${ }^{31}$. Ce rejet d'une certaine tradition littéraire française, Thomas (ainsi que l'auteur?) le fera progressivement sien, ébranlé par les critiques acerbes de Mireille.

Second temps: Thomas d'Amour, influencé par Mireille qui voudrait lui voir écrire un roman policier à l'américaine, renonce à son projet initial, quitte la peau d'Imroul Kais dans laquelle il s'était projeté jusque là et revêt le costume de Justman, personnage de bandit sympathique qui ne vole que les riches. Sur cette lancée, il entreprend un second roman, une variation sur le modèle du roman d'aventures à l'américaine qui subit également les foudres de Mireille: «Thomas d'Amour, lui dit-elle, tu t'es approché de moi en habillant Imroul du costume de Justman, mais tu cherches toujours une pureté importée, comme le yaourt, que tu pourrais déguster à la petite cuiller ! Imroul, traversant à cheval les frontières d'Europe, le sabre au clair, et ton fantôme poursuivant dans les ruelles de Chicago, où le Coca-Cola coule de source, le serpent qui s'est glissé sous le fil barbelé de l'Amérique promise I Arrive en ville! l'Auteur! c'est pas toute de jouer les explorateurs, Tarzan d'Amour! Tu vas t'acheter une carte, le Puni, tu vas débarquer de ta Supernova, tu vas atterrir sur mes prélats fleuris, au son des chants de nos talents Catelli, au soleil de ma Gaspésie, tu vas cultiver nos pissenlits, tes moulins à vent seront des bouteilles en plastique montées sur des piquets de clôture \ $^{32}$

Troisième temps : Thomas d'Amour devient, enfin, un écrivain québécois, se projetant dans le personnage d'un Tarzan original, fils * de Lord Durhan et 
de Jeanne-Mance» 33 et son roman sera pris en charge par Mireille qui, de personnage passif qu'elle était en tant que secrétaire, devient personnage actif, co-auteur: «après souper, dit-elle à Thomas, nous avons un chapitre à écrire épis cette fois je vais le dicter, toi tu vas dactylograpier, chacun son tour à l'aviron sacrement " 34 . Au terme de sa prise de conscience, Thomas a compris où étaient ses racines: «nous, on fait partie de ce petit peup/e, qui a décidé d'habiter ce pays, d'aimer la neige, le froid, la chaleur humide, les saisons inattendues, les volte-face, un petit peuple que l'Histoire, tu sais, celle qu' on nous enseignait à l'école, avait complètement oublié» ${ }^{35}$ et il propose à Mireille de former avec lui une «cellule amour ${ }^{36}$.

C'est par ce biais que sont intégrés dans le récit les événements d'octobre et que l'idéologie contre-culturelle est mise en place (essentiellement dans les communiqués de la cellule amour). Cette idéologie, on le sait, repose sur une prise de conscience planétaire des nouveaux enjeux d'une époque perçue comme subissant une profonde mutation (communiqué numéro 1), sur une conception unanimiste de la société: tout le monde il est beau, tout le monde il est gentil, sauf quelques pourris (communiqué numéro 2), sur l'exaltation de la jouissance immédiate de l'instant présent (communiqué numéro 3), sur une vision anarchisante, anti-étatiste de l'action politique (communiqué numéro 4), sur la mise en évidence des vertus propres au désintéressement: le don (communiqué numéro 5) et l'amour (communiqué numéro 6), sur la célébration du bonheur (communiqué numéro 7) et de la future communauté universelle: la terre comme formant une seule et grande famille ${ }^{37}$. C'est par là, on le voit, que le roman effectue une avancée, en intégrant l'idéologie contre-culturelle d'origine américaine qui prend, dans le contexte québécois, une coloration bien particulière et qui assure au néo-nationalisme un caractère progressiste et ouvert. Mais, bien entendu, le nationalisme demeure l'idéologie de base du récit: il assimile en l'accommodant le discours contre-culturel.

Par ailleurs, si Thomas s'est "converti”, est devenu écrivain québécois, c'est grâce à Mireille dont le roman est aussil'histoire d'amour, racontant en somme les retrouvailles du peuple (Mireille) et de la petite bourgeoisie intellectuelle (Thomas). Ce qui est implicite, c'est que Thomas, finalement, est aussi du peuple, cette partie saine de la nation. Si durant un temps il s'en est séparé, c'est qu'il était sous l'influence étrangère, soit de la France, soit des U.S.A. ${ }^{38}$.

A la fin du récit, il semble admettre avec Mireille que «le langage est une richesse naturelle nationale, comme l'eaus ${ }^{39}$ dont l'écrivain n'a pas le droit d'user pour son seul profit et sa seule jouissance, qu'il n'y a pas, si l'on pousse la logique jusqu'au bout, d'écrivain puisque aussi bien tout le monde peut l'être. Si Galarneau passait d'une conception de l'écriture comme outil au vécrire. Thomas, lui, passe de la pratique d'une écriture désincarnée, décadente à la pratique d'une écriture vivante, populaire (bien collectif) mettant en cause son image et son statut - faux - d'écrivain.

On aura remarqué qu'en un sens $d^{\prime} A$ mour $P . Q$. fournit une image condensée, synthétique du trajet de l'œuvre depuis le début. Dans le person- 
nage de Thomas, aux différents moments de son évolution, on peut retrouver en effet les héros des ceuvres antérieures: le narrateur de l'Aquarium aux prises avec ses émois existentiels, celui du Couteau sur la table à la recherche de son identité personnelle (et collective), celui de Salut Galarneau! se reconnaissant comme Canadien français nationaliste, mais non indépendantiste, n'ayant pas encore tiré les conséquences politiques de la découverte de son identité nationale. A un autre niveau, on peut mesurer le trajet parcouru par l'auteur depuis une conception initiale du roman s'apparentant à celle du «nouveau roman» français à la conversion au «roman québécois» en passant par l'étape intermédiaire du roman de «la francité». Vu sous cet angle, $D^{\prime}$ Amour $P . Q$. est un roman-charnière: par certains côtés (notamment l'intégration de la contre-culture) il annonce le passage à une nouvelle problématique: par d'autres (et ils sont les plus nombreux) il apparaît comme l'aboutissement (et le résumé) de la problématique nationaliste mise en forme, de manière implicite, dès I'Aquarium.

Avant de passer à l'analyse de I'/sle au Dragon, il n'est sans doute pas inutile de faire très rapidement le point. De $L^{\prime} A q u a r i u m$ à $D^{\prime} A m o u r P . Q$. la production de Godbout se laisse aisément regrouper en deux sous-ensembles (les romans du problématique et les romans de l'affirmation) à l'intérieur d'un processus plus large dont l'axe est la recherche d'une identité (personnelle et collective). Romans du problématique, les deux premiers récits le sont en ce qu'ils posent comme enjeu central la quête de valeurs authentiques (pouvant donner un sens à la vie) par des héros jeunes cherchant leur voie au moment d'entrer dans l'âge adulte. Cependant cette quête n'est pas abstraite: elle se déroule à un moment et en des lieux bien précis, dans I'Aquarium en milieu colonial au moment où l'empire - auquel appartient objectivement le héros - s'écroule, dans Le couteau sur la table en milieu canadien agité par la question nationale que pose l'existence du peuple canadien-français. Si, dans le premier récit, la problématique nationaliste est abordée en quelque sorte de l'extérieur, dans le second elle l'est de l'intérieur et oblige le héros, après une longue hésitation, à faire un choix, à choisir son camp (à l'époque, il est important de le souligner, de la première vague felquiste). Si les héros de ces deux premiers récits sont véilléitaires, irrésolus, le plus souvent passifs, il n'en va pas de même pour ceux des romans qui suivent. François Galarneau exprime par sa joie de vivre la santé et la vitalité du peuple québécois et le récit ne raconte plus l'histoire d'une conscience dévorée par le doute mais bien celle d'une naissance: l'accouchement d'un écrivain authentiquement populaire qui saura se faire un juste écho - caisse de résonance - de l'âme collective. Thomas d'Amour, de même, écrivain "pogné» à l'origine se transforme grâce à Mireille en écrivain libéré capable de se faire le chantre éloquent de la québécitude, de la spécificité culturelle et nationale québécoise. Ainsi ces deux romans ne traduisent plus tant une recherche problématique d'une identité qu'une reconnaissance, une affirmation (défense et illustration) de celle-ci. Reste qu'il y a entre ces quatre 
productions des différences - je les ai soulignées au cours de l'analyse qui, à mon sens, témoignent d'une évolution de la conjoncture culturelle et politique du Québec et trouvent en celle-ci leur raison d'être. C'est ce que je tenterai de faire ressortir à la suite de l'étude de l'/s/e au Dragon.

\section{L'Isle au dragon : dépassement de la problématique nationaliste}

En 1976, Godbout publie L'/s/e au dragon qui marque un tournant dans son ceuvre. Le récit, cette fois, n'est plus dédicacé à un individu (Nadeau ou Duguay) ni à une petite communauté (les rédacteurs de Liberté) mais à une collectivité large, aux "vrais insulaires", c'est-à-dire à tous ceux qui voudront bien se reconnaître dans la situation des habitants de l'Isle au dragon, de même qu'à la "princesse Rubis", personnage fabuleux dont la possession sera la récompense de la quête du héros. Par cette indication, l'auteur place d'entrée de jeu son récit sous le signe de la fable, de l'allégorie, du conte, genre dont i) respectera scrupuleusement les règles dans la suite du texte - en empruntant non seulement la structure d'ensemble, mais aussi les détails d'articulation - qui, ainsi, se prêterait aisément à un décodage inspiré par la procédure propienne. Cependant, je ne me livrerai pas à ce travail qui présente son intérêt, préférant concentrer mon attention sur la lecture politique qu'on peut faire du roman.

Sur le plan structurel, on constate une reprise de la situation de base des deux premiers romans : à nouveau un héros est coincé entre deux univers antithétiques, celui des colonisés, des insulaires dont il fait partie, celui des colonisateurs, dirigeants des grandes firmes multinationales représentées par le personnage de Shaheen. Plus précisément, la structure du roman apparaît comme une sorte de décalque de celle du Couteau sur la table puisque le héros appartient à l'univers des colonisés let non à celui des colonisateurs comme c'était le cas dans ('Aquarium), univers dans lequel il s'éprouve comme minoritaire, marginal, entretenant avec sa communauté des rapports qui ne vont pas de soi, qui posent probleme. Mais du Couteau sur la table (1965) à $L$ 's/e au Dragon (1976) il y a tout de même, sur fond de cette ressemblance, une différence essentielle, le colonisateur, f'ennemi principal n'étant plus le Canada anglais mais le grand capital international, et au premier chef l'impérialisme américain, substitution qui, bien entendu, est d'une importance capitale.

L'ennemi du héros est en effet une multinationale, la Pensylvania and Texas International (P.T.I.), pieuvre aux mille tentacules dont le président directeur-général ne «connaît pas, ce qui est normal, toutes les opérations de toutes les filiales, car chacune comme un courant de fraisier prend racine et fait des plans autonomes»39, immense empire rayonnant sur les cinq continents accomplissant aussi bien, et tout à la fois, une œuvre de vie et une œuvre de mort, reconstruisant ici ce qu'elle détruit là (faisant de l'écologie même une industrie). Au Québec, et plus précisément à l'Isle Verte, c'est un Dépotoir atomique contrôlé (un D.A.C.) que la P.T.I. entend installer, ayant choisi ce site pour sa beauté car ainsi que le confie son P.D.G. au héros «c'est 
par la poésie que nous choisissons originellement les lieux d'emplacement de nos installations» ${ }^{40}$, expliquant que son entreprise, comme les autres multinationales d'ailleurs, n'est pas insensible à l'environnement, finançant même des instituts de recherche et des facultés à cette fin, soumettant ainsi les sciences humaines - la psychologie, la sociologie - et même la littérature à ses objectifs. Les multinationales sont donc plus que des entreprises économiques; elles exercent, par des pressions discrètes mais décisives grâce à leur apport aux caisses électorales, un pouvoir politique et, plus profondément encore, elles imposent à la société un moule idéologique conforme à leurs intérêts. Le mot de Marx n'a jamais été plus juste qu'aujourd'hui: les idées dominantes, dans une société, sont celles de la classe dominante, du groupe qui sait assurer son hégémonie dans toutes les dimensions de la vie sociale, même dans celles qui apparaissent relever le plus directement de la vie personnelle (comme, par exemple, les valeurs). Ces multinationales, par ailleurs, sont tout a la fois le produit et un facteur essentiel de production let de reproduction) de "L'Empire de l'asphalte " 41 , de la "civilisation du compte en banque» dont le $\alpha$ centre d'achats est le véritable centre culturel» ${ }^{42}$, civilisation qui, grâce à elles, étend progressivement sa domination sur l'ensemble de la planète ${ }^{43}$.

Ce monstre à plusieurs têtes, ce dragon maléfique est représenté concrètement par le personnage de Shaheen, incarnation par excellence du «mythe américain» du petit gars d'origine modeste qui, à force de travail et de volonté, finit par se hisser à la direction d'un empire, assumant à cinquante ans "la gérance d'un complexe financier pour lequel les frontières nationales ne sont que constructions théoriques, cure-dents qu'on envoie promener d'une chiquenaude sur la table défaite entre la poivrière et le cendrier ${ }^{44}$. C'est à cet homme, instrument d'une énorme puissance, que le héros devra s'affronter.

En face de cet empire, objet de sa convoitise: la petite communauté de l'Isle Verte. Celle-ci, séduite par les offres financièrement alléchantes de Shaheen, accepte la rage au cceur, mais accepte tout de même de vendre l'Isle et de s'en retirer, cédant à un raisonnement fort prosaïque: avec cet argent nous pourrons, ailleurs, vivre mieux. Ce comportement, bien entendu, est révélateur de l'indice élevé de pénétration idéologique des valeurs et de la culture américaine dans l'Isle (et au Québec). Car si les habitants de l'Isle cèdent aussi facilement aux pressions de Shaheen, c'est qu'ils y sont en somme prêts, leur culture ayant été préalablement détruite par la civilisation Pepsi. De plus, bien entendu, ils ne sont pas défendus par leur gouvernement lui-même sous la coupe des multinationales - qui ne leur propose pas de solution de remplacement. De cette communauté résignée, passive, Shaheen, on s'en doute bien, ne fera qu'une bouchée.

Le héros-narrateur, quant à lui, bien qu'appartenant à l'univers des colonisés, fait figure de cavalier solitaire, étant seul à vouloir se battre farouchement contre la P.T.I. et étant, de la sorte, incompris des habitants de l'isle. Il faut dire qu'il n'est pas, par ses origines, membre de la communauté, qu'il est venu à l'Isle en étranger séduit par son charme et que son intégration 
donc pose problème. On voit qu'à nouveau dans ce récit Godbout soulève la question stratégique des rapports des intellectuels (aux racines souvent peu profondes) avec le groupe auquel ils s'identifient, sans forcément lui appartenir «naturellement». Dans $L \%$ sle au dragon, le héros ne réussit pas à entraîner l'adhésion de la communauté à ses thèses (qui s'inspirent d'une logique autre que celle de la rationnalité capitaliste dont les habitants de l'Isle sont imprégnés) et c'est en solitaire qu'il devra combattre le monstre.

Sa lutte sera, par conséquent, celle du poète et de l'homme d'affaires. Contrairement toutefois à ce qui se passe dans la réalité, c'est le poète qui sera victorieux, qui neutralisera le dragon et obtiendra en récompense «la princesse (l'adhésion de la communauté?). Tout finit donc bien, ce qui est logique dans l'univers du conte, où le héros toujours triomphe de l'anti-héros, mais qui ne va pas du tout de soi dans la réalité. Or, le roman de Godbout, évidemment, vise, au-delà de la fable, à faire prendre conscience d'un problème réel: la percée envahissante du capital américain dans toutes les sphères d'activité (autant culturelle qu'économique) du Québec (dont l'Isle est aussiune figure), le Québec qui, en cela est dans une situation de subordination, de dépendance - et d'opposition - analogue à celle de l'Irlande par rapport aux Anglais et de la Tchécoslovaquie par rapport aux Allemands ou aux Russes. Les Québécois, dans cette perspective évoquée par le récit, sont les Irlandais et les Tchèques de l'Amérique du Nord et, pour autant, se trouvent dans une position extrêmement délicate face à leur puissant voisin et ennemi.

On aura remarqué que cette donnée centrale, la pénétration des multinationales, entraîne un déplacement de la problématique qui soustend les romans de Godbout. La recherche d'identité nationale, puis son affirmation, par rapport à la domination anglo-saxonne n'assure plus la structure de base du récit. Cependant il serait hasardeux d'affirmer que nous sommes en présence d'un renversement total de la problématique dans la mesure où la défense de l'Isle s'identifie d'abord à la lutte pour la préservation de l'identité culturelle québécoise (et de son patrimoine) et où la question de la domination impérialiste n'est pas résolument posée sur le terrain économique. Reste qu'on ne saurait nier que la problématique a subi un déplacement significatif dont le principe, à nouveau, nous renvoie à l'analyse de la conjoncture.

1. «Ce texte constitue la première partie d'une étude de l'œuvre de Jacques Godbout à paraître dans un livre en préparation: Le roman québécois contemporain: une lecture politique".

2. André Smith, L'Univers romanesque de Jacques Godbout, Editions Aquila, 1976. p. 6 (Coll. Figures du Québec).

3. Idem, p. 79.

4. L'Aquarium, Paris, Editions du Seuil, 1962, p. 108.

5. L'action du roman se situe à la fin de la troisième période de l'histoire coloniale. dans la typologie suggérée par Jacques Leenhardt: durant la première période de cette histoire (1860-1920) qui est d'abord une conquête c'est le colon pionnier qui est la figure centrale; durant la seconde période (1920-1945) qui est mise en valeur de la conquête c'est l'exploiteur colonial qui est la figure dominante; enfin, 
durant la dernière période (1945-1960), celle de la décolonisation, c'est le coopérant qui apparaît comme le type principal (re. Jacques Leenhardt, Lecture politique du roman, Paris, Editions de Minuit, 1973, p. 159 et ssq.).

6. L'Aquarium, p. 18.

7. Idem, p. 24.

8. Idem, p. 69.

9. L'assimilation des habitants de la casa à des escargots est sans doute la comparaison qui est reprise le plus souvent dans le roman pour décrire l'humanité sans espoir et veule qui caractérise les individus d'une période irrémédiablement enfoncée dans le déclin et la décadence.

10. L'Aquarium, p. 22.

11. Idem, p. 62.

12. Idem, p. 106.

13. Idem, p. 22.

14. L'anticléricalisme est une constante dans tous les romans de Godbout. Virulent au début, il s'atténuera dans les derniers romans sans disparaître complètement. Ce n'est pas là la seule constante, bien entendu, de cette œuvre. Dans tous les romans on retrouve au niveau thématique, outre l'anticléricalisme, une profonde nostalgie de l'enfance et de l'adolescence, au niveau des personnages la mise en scène d'un type de héros bien précis: jeune homme de la petite-bourgeoisie québécoise aux prises avec des interrogations "philosopiques", des émois amoureux et des rapports - a définir et établir - avec la société, au niveau structurel un récit fragmenté, brisé dont Gilles Marcotte a proposé une très pertinente description dans Le roman à l'imparfait, Montréal, La Presse, 1976. pp. 139-169. Ce sont ces constantes qui caractérisent dans une large mesure la manière de Godbout, le style (entendu au sens d'organisation d'ensemble du texte) qui le distingue des autres écrivains de sa génération et notamment d'Hubert Aquin.

15. Lire, entre autres, le passage particulièrement révélateur de la page 56 du roman.

16. Le couteau sur la table, Paris, Éditions du Seuil, 1965, p. 9.

17. Idem, p. 103.

18. Les U.S.A. sont représentés à la fois comme puissance militaire - dans les extraits de journaux insérés dans le roman - la plus importante du monde, et comme nouveau modèle de civilisation, la société post-industrielle ayant dépassé le stade de la production pour satisfaire des besoins, parvenue à un niveau de développement tel qu'il faut, pour en assurer le fonctionnement, créer de nouveaux besoins tout à fait artificiels: société du gadget fondée sur la fabrication de l'éphémère et de l'inutile. II s'agit là d'un thème que l'on retrouvera sous diverses formes dans les romans ultérieurs.

19. Le couteau sur la table, p. 50.

20. Idem, p. 57.

21. Idem, p. 71.

22. Idem, p. 88.

23. Le héros écrit, page 144, "Ce n'est pas tellement l'âge aujourd'hui qui m'épuise: ni de si peu faire l'amour (cela me porte bien sûr à des tristesses excessives), mais à tourner en rond autour du puits sans jamais y pouvoir plonger, me chagrine $(. .$. En fait je me sens plus malade que vieux». Je souligne.

24. Lire le passage auto-accusatoire particulièrement virulent des pages 85 et 86 de Salut Galarneau/ Paris, Éditions du Seuil, 1967.

25. Idem, p. 73.

26. Idem, p. 125.

27. Idem, p. 106.

28. Mireille, regardant Thomas lorsqu'elle le rencontre pour la première fois, lui trouve "l'ceil soucieux (...) l'air qu'on porte comme des lunettes au Département d'études françaises " et elle songe qu'avec sa «gueule triste", il pourrait « diriger un séminaire au Département „. D'Amour P.Q. Paris, Éditions du Seuil, 1972, pp. 67 et 68 . L'ironie à l'endroit des professeurs en tous genres, et surtout des professeurs de littérature 
et de sociologie, est un trait récurrent de l'ceuvre de Godbout. Cette ironie est particulièrement féroce lorsqu'elle s'applique aux «sociologues» qui autant dans Salut Galarneau, dans $D^{\prime} A$ mour $P . Q$. que dans $L '$ 's/e au dragon apparaissent comme des distilleurs d'évidences, des faux savants quand ils ne sont pas représentés, tout simplement, comme des mercenaires du Capital. A quoi tient cette agressivité est bien entendu une autre question à laquelle il est bien difficile de répondre; aussi je me bornerai à la signaler.

29. Idem, p. 34

30. Idem, p. 16.

31. Idem, p. 24.

32. Idem, p. 113

33. Idem, p. 119

34. Idem, p. 116.

35. Idem, p. 126. Je souligne cette expression si typiquement «lévesquiste».

36. Idem, p. 127.

37. On aura reconnu là les grands traits du discours utopiste tels que les a dégagés notamment Jean Servier dans son Histoire de l'utopie, Paris, Gallimard, 1967 (Coll. ldées).

38. Sur ce point, il faudrait citer le long passage de la page 124 sur les * obstacles » que franchissent en moto Mireille et Thomas: murs incarnant respectivement la tradition judéochrétienne, la civilisation française décadente, la société américaine du gadget.

39. L'/s/e au Dragon. Paris, Éditions du Seuil, 1976, p. 21.

40. Idem, p. 61.

41. Idem, p. 48.

42. Idem, p. 129 .

43. Dans le roman, Godbout donne l'exemple du cinéma qui a su créer et imposer à l'admiration du monde (à la rêverie des adolescents de tous les continents) ces créatures mythologiques que sont les stars au premier rang desquelles Marylin Monroe qu'a connue et rêvé de posséder son héros lors du tournage d'un film au lac Louise au début des années 1960 . On pourrait aussi invoquer l'exemple de la musique, l'Amérique ayant réussi à imposer ses rythmes à la jeunesse du monde entier, et de manière plus générale tous les phénomènes relevant de la culture populaire.

44. L\%sle au dragon, p. 23. 